\title{
BEAM-COLUMN RESISTANCE ACCORDING TO SLOVAK STANDARD AND EUROCODES
}

The article deals with the verification of resistance of torsionally restrained hot-rolled beam-columns of $H$ or I cross-sectional shape and also of the perpendicular hollow cross-section subjected to the biaxial bending and axial compression. Results of analysis of the beam-column resistances according to methods of former STN 731401 [1] and the current STN EN 1993-1-1 [2] are compared with results of numerical approaches. The observed members are subjected to the normal force and to the transverse loads uniformly distributed along the length of both axes. The cross-sections of HEB 300, IPE 300 and RHS 300x200x10 were chosen to compare obtained results.

Keywords: Axial force, bending moment, torsional restraint, beam-column.

\section{Introduction}

Nowadays, the development of new materials creates a competitive environment for the use of steel as a structural material in constructions. High-strength concrete and reinforced concrete cross-sections of high quality compete with steel with their dimensions. Therefore, many specialists are searching to improve the accuracy of design formulas for steel members, especially for members subjected to the combined actions. The beam-column subjected to combination of bending moments and axial compression is an example of this problem. Therefore, the possibility of using cross-sectional plastic reserve or improving and more specifying the design approaches to verification of beam-column resistance becomes more important. If the standard prescriptions do not meet criteria of the optimal structural design, then the determination of the structural resistance and its verification need necessarily to be more precise and often complex based on the numerical calculations using FEM models.

\section{Analysis of beam-column behaviour}

\subsection{Analytical approach}

Stability analysis of a beam-column has been done in the past using solution of the relevant homogeneous differential equations. The buckling resistance of initially deflected beam-column with double symmetric constant cross-section, subjected to combined constant axial compression and biaxial bending due to transverse load uniformly distributed along the length of the both axes, could be described by the system of inhomogeneous differential equations in accordance with [3]

$$
\begin{aligned}
& E I_{y} w^{(4)}+N\left(w+w_{o}\right)^{\prime \prime}+\left[M_{z}\left(\theta+\theta_{o}\right)\right]^{\prime \prime}=q_{z} \\
& E I_{z} v^{(4)}+N\left(v+v_{o}\right)^{\prime \prime}+\left[M_{y}\left(\theta+\theta_{o}\right)\right]^{\prime \prime}=q_{y} \\
& E I_{w} \theta^{(4)}-G I_{t} \theta^{\prime \prime}+i_{s}^{2} N\left(\theta+\theta_{o}\right)^{\prime \prime}+ \\
& +\left[q_{y} z_{g, y}+q_{z} z_{g, z}\right]\left(\theta+\theta_{o}\right)+\left[M_{y}\left(v+v_{o}\right)^{\prime}\right]^{\prime}+ \\
& +\left[M_{z}\left(w+w_{o}\right)^{\prime}\right]^{\prime}=0
\end{aligned}
$$

In the case of the torsionally restrained beam-column along its length, i.e. the member whose effects of lateral-torsional buckling are eliminated by the relevant supporting, and considering the initial deflection in the direction of the y-y axis only, the system of differential equations (1) can be rewritten into the following elementary pair of independent differential equations, describing the buckling resistance of beam-column respecting the above mentioned cross-sectional shape and load

$$
\begin{aligned}
& E I_{y} w^{(4)}+N w^{\prime \prime}=q_{z} \\
& E I_{z} v^{(4)}+N\left(v_{o}^{\prime \prime}+v^{\prime \prime}\right)=q_{y}
\end{aligned}
$$

where

E is the Young's modulus of elasticity,

$G \quad$ is the shear modulus,

$I_{y}, I_{z} \quad$ is the second moment of area about the y-y or z-z axis, respectively,

$I_{t} \quad$ is the St. Venant torsional constant,

\footnotetext{
* Peter Janik, Josef Vican

Department of Structures and Bridges, Faculty of Civil Engineering, University of Zilina, Slovakia,

E-mail: peter.janik@fstav.uniza.sk
} 


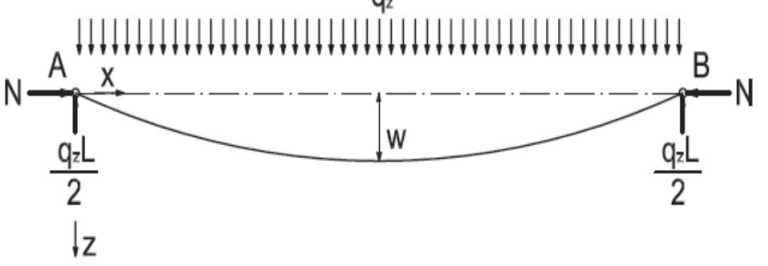

$q_{2} x$

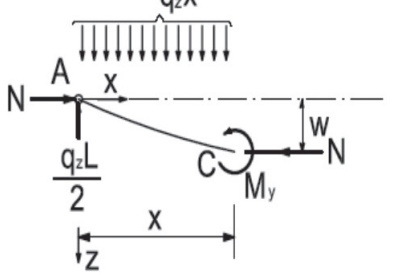

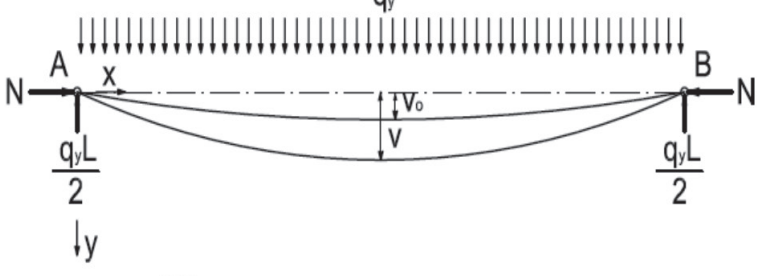

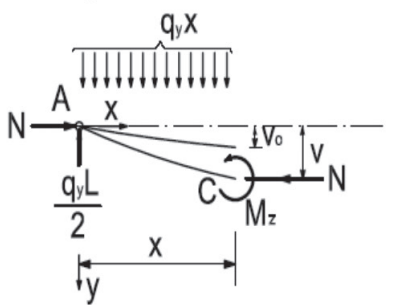

Fig. 1 Simply supported beam-column subjected to the constant axial compression and uniformly distributed transverse load

$I_{w} \quad$ is the warping torsional constant,

$N$ is the normal compression force,

$M_{y}, M_{z}$ are the bending moments about the y-y and z-z axis, respectively, induced by transverse loads,

$i_{s} \quad$ is the cross-sectional polar radius of gyration around to the centre of shear,

$q_{y}, q_{z} \quad$ are the transverse uniformly distributed loads in the direction of the $y-y$ or z-z axis, respectively,

$v_{o} \quad$ is the initial deflection of a member in the direction of the y-y axis,

$v \quad$ is the deflection increment of a member in the direction of the y-y axis,

$w \quad$ is the deflection increment of a member in the direction of the $\mathrm{z}-\mathrm{z}$ axis,

$w_{0} \quad$ is the initial deflection of a member in the direction of the $\mathrm{z}-\mathrm{z}$ axis,

$z_{g, y}, z_{g, z}$ are the distances of the applied transversal load from the centre of shear measured in the direction of the $y-y$ or z-z axis, respectively,

$\theta_{0} \quad$ is the angle of the initial cross-sectional rotation of a member about the $\mathrm{x}-\mathrm{x}$ axis,

$\theta \quad$ is the angle increment of cross-sectional rotation of a member about the $\mathrm{x}-\mathrm{x}$ axis.

Bending moments $\mathrm{M}_{\mathrm{y}}$ and $\mathrm{M}_{\mathrm{z}}$ depend on the type of transverse load and usually have the non-constant shape. According to Fig. 1 , the bending moments at the point $\mathrm{C}$ at a distance of $\mathrm{x}$ from A (see Fig. 1) can be obtained by taking moments about $\mathrm{C}$ for the segment of member A-C using formulas

$$
M_{y}=N w+\frac{q_{z} L x}{2}-\frac{q_{z} x^{2}}{2}
$$

and

$$
M_{z}=N v+N v_{o}+\frac{q_{y} L x}{2}-\frac{q_{y} x^{2}}{2}
$$

The equations become even more inhomogeneous when the initial imperfections are implemented into the system. The member initial deflection of $v_{0}$ in the direction of the $\mathrm{y}$-y axis was considered to simulate the shape of the member equivalent geometrical imperfection described by means of the sinusoidal function as follows

$$
v_{0}=e_{0, z} \sin (\pi x / L)
$$

where the amplitude $e_{0, z}$ is defined according to the standard [2] in the following form

$$
e_{0, z}=\alpha(\bar{\lambda}-0,2) W_{e l, z} / A
$$

and the imperfection factor $\alpha_{l}$ depends on the cross-sectional shape.

Using the differential expression for bending moment, the equations (3) can be rewritten into the differential form [4] as follows

$$
\begin{aligned}
& w^{\prime \prime}+\mu_{1}^{2} w=\frac{q_{z}}{E I_{y}}\left(\frac{x^{2}}{2}-\frac{x L}{2}\right) \text { with } \mu_{1}^{2}=\frac{N}{E I_{y}} \\
& v^{\prime \prime}+\mu_{2}^{2} v=\frac{q_{y}}{E I_{z}}\left(\frac{x^{2}}{2}-\frac{x L}{2}\right)-\mu_{2}^{2} e_{o, z} \sin \left(\frac{\pi x}{L}\right) \\
& \text { with } \mu_{2}^{2}=\frac{N}{E I_{z}}
\end{aligned}
$$

The final solutions of the above described differential equations can be obtained using homogenous boundary condition, $\mathrm{w}=\mathrm{v}=0$ for $\mathrm{x}=0$ and for $\mathrm{x}=\mathrm{L}$, in the form as follows

$$
\begin{aligned}
& w=\frac{q_{z}}{N \mu_{1}^{2}}\left(\tan \frac{\mu_{1} L}{2} \sin \mu_{1} x+\cos \mu_{1} x-1\right)+ \\
& +\frac{q_{z}}{2 N}\left(x^{2}-x L\right) \\
& v=\frac{q_{y}}{N \mu_{2}^{2}}\left(\tan \frac{\mu_{2} L}{2} \sin \mu_{2} x+\cos \mu_{2} x-1\right)+ \\
& +\frac{q_{y}}{2 N}\left(x^{2}-x L\right)+\frac{N e_{o, z}}{N_{c r, z}-N} \sin \left(\frac{\pi x}{L}\right)
\end{aligned}
$$


where $N_{c r, z}=\pi^{2} E I / L_{z}^{2}$ is the Euler flexural buckling force.

The displacement at any point of the member can be calculated by inserting appropriate values of $N, q$ and $x$ in these equations. The maximum deflections and maximum bending moment are in the mid-span of the observed beam-column and can be expressed by the following formulas

$$
\begin{aligned}
& w_{\max , y}=\frac{q_{z}}{N \mu_{1}^{2}}\left(\sec \frac{\mu_{1} L}{2}-1\right)-\frac{q_{z} L^{2}}{8 N} \text { and } \\
& M_{\max , y}=\frac{q_{z} E I_{y}}{N}\left(\sec \frac{\mu_{1} L}{2}-1\right) \\
& v_{\max , z}=\frac{q_{y}}{N \mu_{2}^{2}}\left(\sec \frac{\mu_{2} L}{2}-1\right)-\frac{q_{y} L^{2}}{8 N}+\frac{N e_{o, z}}{N_{c r, z}-N} \text { and } \\
& M_{\max , z}=\frac{q_{y} E I_{z}}{N}\left(\sec \frac{\mu_{2} L}{2}-1\right)+\frac{N e_{o, z}}{1-\frac{N}{N_{c r, z}}}
\end{aligned}
$$

Then, the maximum stress in the mid-span of the beamcolumn can be obtained using the relation

$$
\sigma_{\max }=\frac{N}{A}+\frac{M_{\max , z}}{W_{e l, z}}+\frac{M_{\max , y}}{W_{e l, y}}
$$

where $W_{e l, y}, W_{e l, z}$ are the elastic sectional modulus about the y-y or $\mathrm{z}-\mathrm{z}$ axis, respectively.

\subsection{Numerical approach}

Due to the complexity of analytical solutions of system of inhomogeneous differential equations according to (1), the numerical analyses are used to obtain results. Numerical models are usually created using computer software based on FEM. In this case the Ansys-Workbench was used. Numerical model developed in this environment consists of one dimensional finite element BEAM 188 (see Fig. 2). The element is based on Timoshenko's beam theory including shear-deformation effects. The unrestrained warping of cross-section or restrained one respectively can be taken into account. This element is a linear, quadratic, or cubic two-node beam element in 3D. BEAM188 has six or seven degrees of freedom at each node. These include translations in the $\mathrm{x}, \mathrm{y}$, and $\mathrm{z}$ directions and rotations about the $\mathrm{x}, \mathrm{y}$, and $\mathrm{z}$ directions. The seventh degree of freedom - warping magnitude, is optional. This element is well-suitable for linear large rotation and/or large deflection nonlinear applications.

The sinus function was chosen in accordance with [2] to simulate the initial shape of bow imperfection of the member in the direction of the $y-y$ axis. Its amplitude was taken in compliance with recommendation for flexural buckling in [2]. In the case of applied numerical model, the boundary conditions Rot, $\mathrm{x}=0$ were considered, allowing for torsionally restrained member with disabled rotation about the x-axis.

Ideal elastic-plastic material model was chosen. Material characteristics were used according to standard recommendations [2].

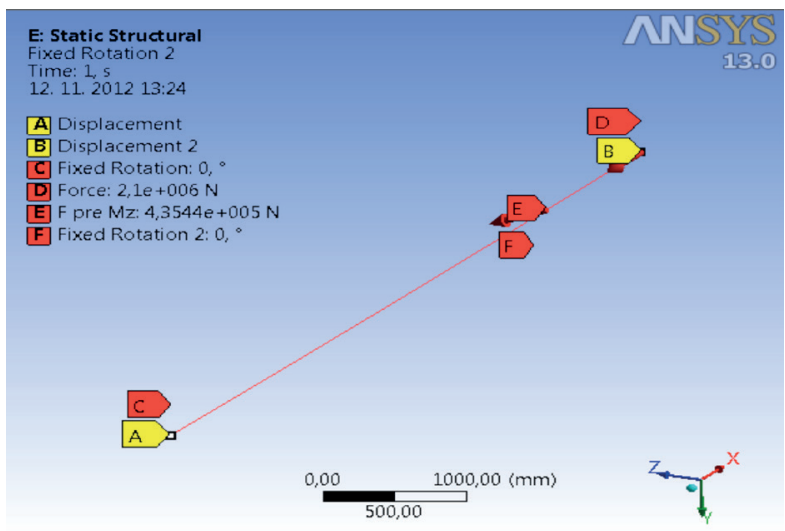

Fig. 2 The numerical model

To verify the described numerical model, the analytical solution of the problem derived in section 2.1 was used. The results of maximum stresses calculated by means of formula (9) and using the above mentioned numerical model for beamcolumn of IPE 300 cross-section having slenderness of $\bar{\lambda}_{z}=1.0$ and 1.5 are compared in Table 1 . The comparison is presented in the form of ratio of results of numerical calculations to results obtained using derived analytical solutions. The comparison presented in Table 1 is statistically evaluated to determine the error of the numerical approach.

Statistically evaluated comparisons of analytical calculations with results of numerical ones

Table 1

\begin{tabular}{|c|c|c|}
\hline \multicolumn{3}{|c|}{ IPE 300} \\
\hline $\bar{\lambda}$ & 1 & 1,5 \\
\hline Mean value & 1.0086 & 1.0087 \\
\hline Maximum value & 1.0160 & 1.0130 \\
\hline Minimum value & 1.0041 & 1.0043 \\
\hline$\Sigma$ calculations & 27 & 27 \\
\hline
\end{tabular}

The comparison presented in Table 1 shows a very good accordance insomuch that above described numerical model can be applied for parametric numerical study of the beam-column resistance determination. It was necessary to evaluate a large number of combinations of normal forces and transverse loads to create compact resistance surface of the observed members. Therefore, the following input values were taken into account for numerical calculations in frame of parametric study:

- yield strength of $235 \mathrm{MPa}$, the Young's modulus of elasticity of $210 \mathrm{GPa}$, the zero modulus of strain hardening and the Poisson ratio of 0.3 ;

- the amplitude of the initial bow imperfection was taken according to formula $e_{o, z}=\alpha_{1}(\bar{\lambda}-0,2) W_{e l, z} / A$ (A is the cross-sectional area of a member) with $\alpha_{1}=0.49$ for HEB 300, $\alpha_{1}=0.34$ for IPE 300 and $\alpha_{1}=0.21$ for RHS $300 \times 200 \times 10$;

- the geometric nonlinearity and torsionally restrained member with condition $\operatorname{Rot}, \mathrm{X}=0$ was considered; 
- cross-sectional characteristics of thin-walled sections were taken according to the table from [5].

\subsection{Standard approach according to STN 731401}

The standard procedures are used for assessment of members to achieve the greater extent. The resistance of member subjected to the combined biaxial bending and axial compression was verified by means of formulas according to STN 731401 [1] The latest revision of this standard was valid from 23.03.1998 This latest version of the standard was applied in our country till 01.04.2010, when it was replaced, as well as the other Slovak structural standards, by Eurocodes.

In the parametric study, the analyses of the resistance of members with cross-sections of Class 1 and 2 were performed. Member not susceptible to torsional deformations with crosssections of Class 1 or 2, respectively, shall satisfy the following single condition in accordance with [1]

$$
\begin{aligned}
& \frac{N_{E d}}{\chi_{\min } N_{R k} / \gamma_{M 1}}+\frac{k_{y} M_{y, E d}}{\chi_{L T} M_{y, R k} \gamma_{M 1}}+ \\
& +\frac{k_{z} M_{z, E d}}{M_{z, R k} / \gamma_{M 1}} \leq 1,0
\end{aligned}
$$

where

$N_{E d} \quad$ is the design compression force,

$M_{y, E d}, M_{z, E d} \quad$ are the design bending moments about the y-y or z-z axis, respectively,

$N_{R k} \quad$ is the characteristic value of the resistance to normal compression force,

$M_{v, R k}, M_{z, R k} \quad$ are the characteristic values of the resistances to bending moments about the $y-y$ or $z-z$ axis, respectively,

$k_{y}, k_{z} \quad$ are the interaction factors,

$\chi_{\min } \quad$ is the minimum of reduction factors due to flexural buckling to $y-y$ or z-z axis, respectively,

$\chi_{L T} \quad$ is the reduction factor for lateral-torsional buckling (in this case $\chi_{L T}=1.0$ ),

$\gamma_{M 1}$

\subsection{Standard approach according to STN EN 1993-1-1}

Set of Eurocodes for the design of building structures has been successively implemented into the STN standard system since 2005. Translations of European standards to individual Slovak ones with the National Annexes were gradually published.

To verify resistance of the member subjected to combination of biaxial bending and axial compression, two methods named $\mathrm{A}$ and $\mathrm{B}$ are possible to apply according to standard [2]. A common form of equations for assessing the resistance of member with cross-section of Class 1 or 2 respectively, subjected to the axial compression and biaxial bending has the form as follows:

$$
\begin{aligned}
& \frac{N_{E d}}{\chi_{y} N_{R k} / \gamma_{M 1}}+k_{y y} \frac{M_{y, E d}}{\chi_{L T} M_{y, R k} / \gamma_{M 1}}+k_{y z} \frac{M_{z, E d}}{M_{z, R k} / \gamma_{M 1}} \leq 1,0 \\
& \frac{N_{E d}}{\chi_{z} N_{R k} / \gamma_{M 1}}+k_{z y} \frac{M_{y, E d}}{\chi_{L T} M_{y, R k} / \gamma_{M 1}}+k_{z z} \frac{M_{z, E d}}{M_{z, R k} / \gamma_{M 1}} \leq 1,0
\end{aligned}
$$

where the following designations are used in addition to ones under relation $(10)$

$k_{i, j}$ are the interaction factors,

$\chi_{y}, \chi_{z}$ are the reduction factors due to flexural buckling to $\mathrm{y}$-y or z-z axis, respectively.

Methods A and B differ from each other by the approach to calculating the interaction factors $k_{i, j}$. Equations for individual interaction factors $k_{i, j}$ were calibrated by means of many geometric and material nonlinear computer simulations (GMNIA) based on the finite element method, [6]. For practical design in Slovakia, the utilization of method B was recommended in the National Annex [7].

\section{Comparison of approaches to verification of beam- column resistance}

The comparison of the results obtained using different standard approaches to results of the numerical calculations is shown in Figs. 3-8. The numerical values represent the size of the third axis in the selected levels of $N_{E d} / N_{R d}$. Ratios $M_{y, E d} / M_{y, R d}$ and

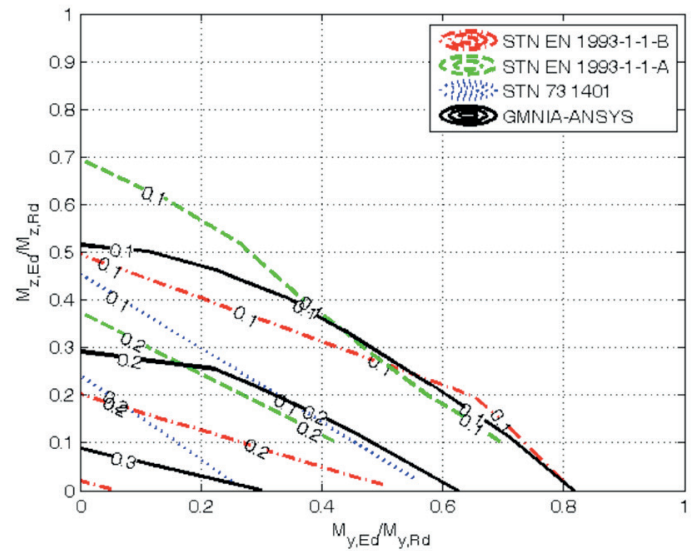

Fig. 3 Comparison of member resistance curves, $\bar{\lambda}_{z}=1.0 ; 1.5-$ HEB 300

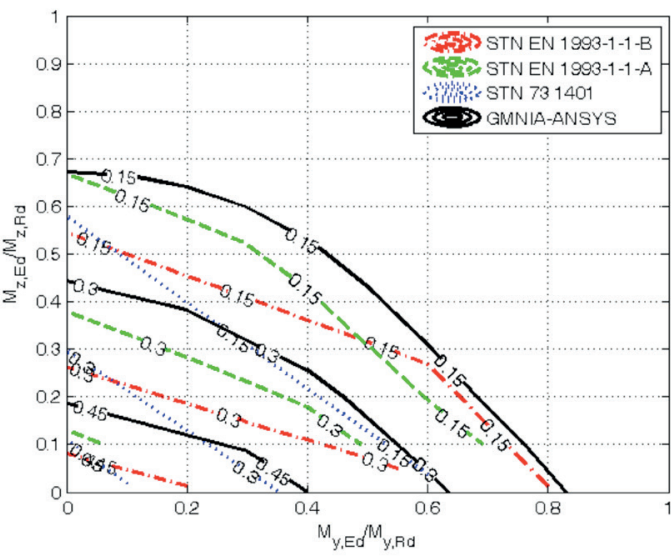


$M_{z, E d} / M_{z, R d}$ are shown on the horizontal and vertical graph axes. Characteristic values of resistances without partial safety factor $\gamma_{M I}$ for material were considered as the standardized member resistances for all normative approaches to compare the obtained results of numerical calculations. Graphs are processed for non dimensional slenderness $\bar{\lambda}_{z}=1.0 ; 1.5$ and 2.0.

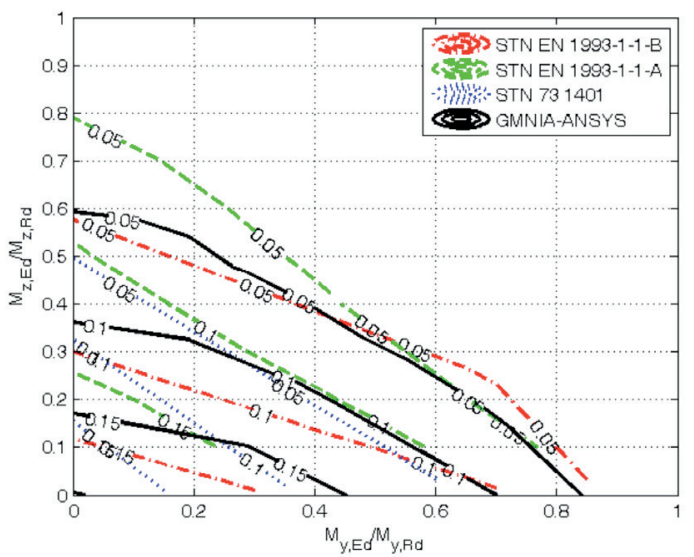

Fig. 4 Comparison of member resistance curves, $\bar{\lambda}_{z}=2.0-$ HEB 300

The investigated member is subjected to the axial compression and bending due to the uniformly distributed transverse load for both axes of symmetry of the cross-section so that the shape of the bending moment is parabolic. Calculations were considered with torsionally restrained cross-section HEB 300 and IPE 300, i.e. the effect of lateral-torsional buckling was neglected. The cross-section of RHS $300 \times 200 \times 10$ is not susceptible to torsional deformations.

\section{Conclusions}

Conservative approach to the resistance verification according to the standard [1] is evident from all the result's

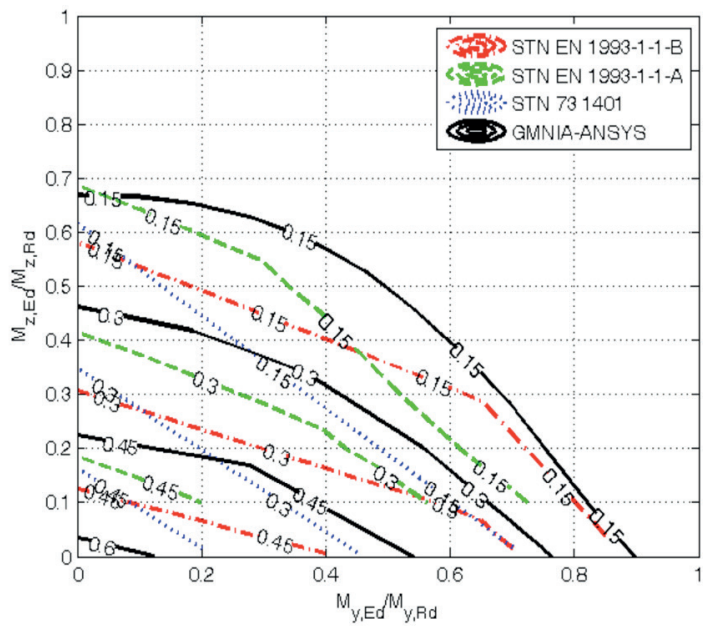

comparisons. In accordance with this standard, the spatial display creates approximately "planar resistance surface" connecting the borderline cases lying on the axes of the graph.

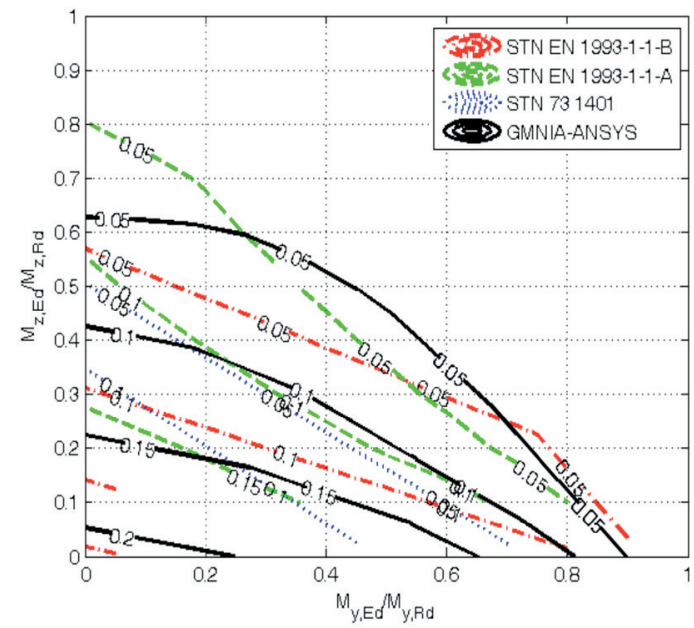

Fig. 6 Comparison of member resistance curves, $\bar{\lambda}_{z}=2.0$ - IPE 300

Resulting isoclines of methods A and B show that the standard [2] uses the plastic reserve of members more accurately. Surface resistance of these approaches is already convex describing more realistic beam-column resistance. Both methods for assessing the combination of biaxial bending and axial compression according to [2] describe the resistance of member with the closed crosssection of RHS 300x200x10 better. In the cases of the higher levels of normal forces, standardized approaches show reserve of resistance compared to results of numerical calculations. If members are subjected to the smaller bending moment $M_{y}$, the method A provides higher levels of the member resistance for non-dimensional slenderness $\bar{\lambda}_{z}$ greater than 1.0 than numerical calculations for open cross-sections IPE 300 and HEB 300. Assessments of members with torsional restraints according to method A are less suitable for members with non-dimensional

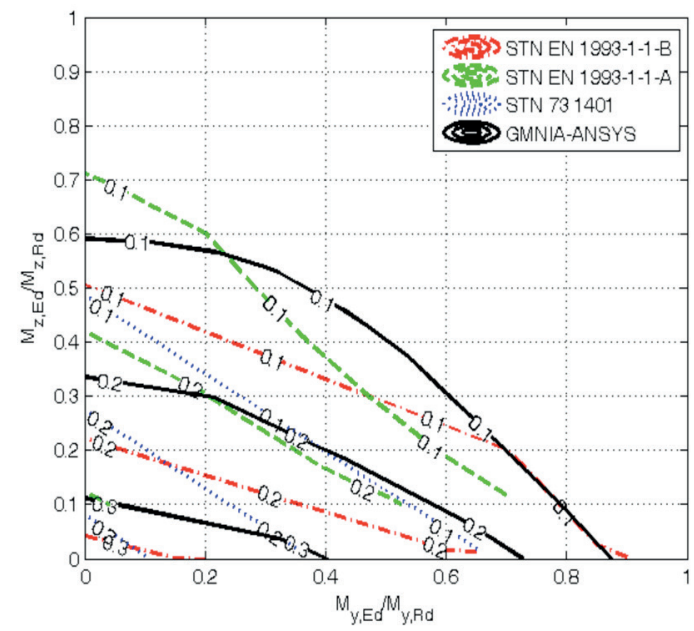

Fig. 5 Comparison of member resistance curves, $\quad \bar{\lambda}_{z}=1.0 ; 1.5$ - IPE 300 

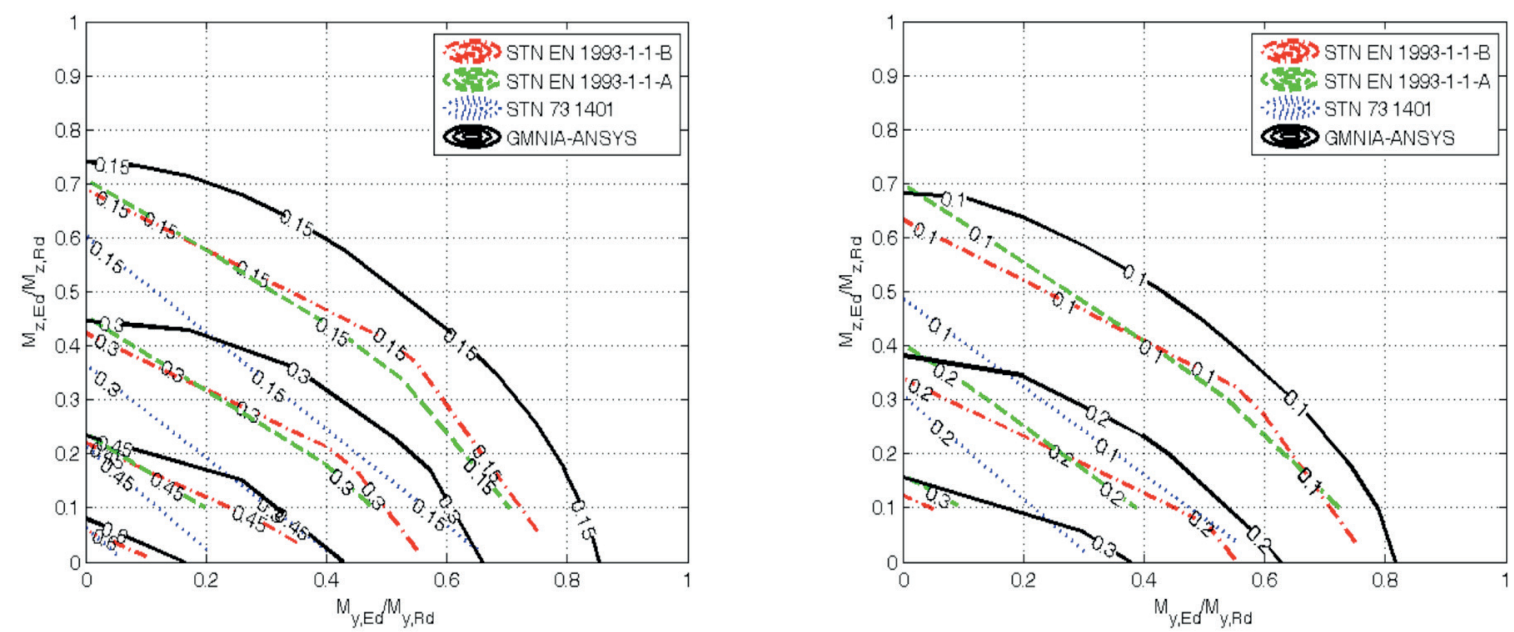

Fig. 7 Comparison of member resistance curves, $\bar{\lambda}_{z}=1.0 ; 1.5-$ RHS 300x200x10

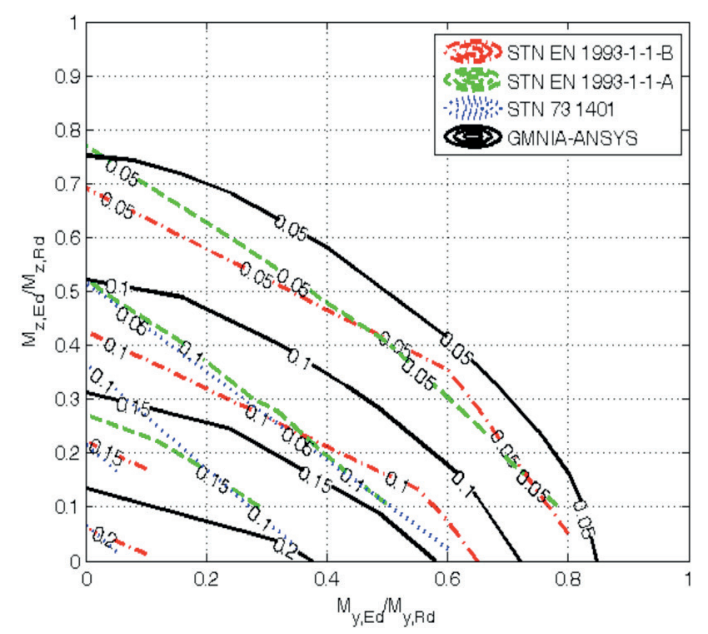

slenderness $\bar{\lambda}_{z}$ greater than 1.0. The recommendation to use the method B in Slovakia seems to be correct from this point of view [8].

\section{Acknowledgements}

This paper presents results of works supported by the Slovak Research and Development Agency under the contract No. APVV-0106-11 and by the Scientific Grant Agency of the Slovak Republic under the project No. 1/0364/12.

Fig. 8 Comparison of member resistance curves, $\bar{\lambda}_{z}=2.0-$ RHS $300 \times 200 \times 10$

\section{References}

[1] STN 731401: Design of Steel Structures. Bratislava, SUTN, 1998.

[2] STN EN 1993-1-1: Design of Steel Structures - Part 1-1: General Rules and Rules for Buildings. Bratislava, SUTN, 2006.

[3] BREZINA, V.: Buckling Strength of Structural Steel Members, Prague, SNTL, 1963, 384 p.

[4] ALLEN, H. G. et al.: Background to Buckling, McGraw-Hill Book Company (UK) Limited. 1980, 582 p.

[5] http://www.staticstools.eu

[6] BOISSONNADE, N.: Rules for Member Stability in EN 1993-1-1, Background documentation and design guidelines. ECCS 2006, $259 \mathrm{p}$.

[7] STN EN 1993-1-1/NA: Design of Steel Structures. Part 1-1: General Rules and Rules for Buildings, Bratislava, National Annex, SUTN, 2006.

[8] VICAN, J., ODROBINAK, J, GOCAL, J., HLINKA, R.: Design and Stability of Long Span Railway Arch Bridge, Communications - Scientific Letters of University of Zilina, No. 4, 2013 (in print). 